\title{
Identificación de Variables Ocultas y su Vinculación con el Reco- nocimiento de la Aleatoriedad ${ }^{1}$
}

\section{Identification of Hidden Variables and their Connection with the Recognition of Random}

\author{
Horacio Félix Attorresi², Alcira Myriam García Díaz y Héctor Omar Pralong \\ Instituto de Investigaciones de la Facultad de Psicología de la Universidad de \\ Buenos Aires, Argentina.
}

(Recepción: Agosto 2009 - Aceptación: Diciembre 2009)

\begin{abstract}
Resumen
Se estudió la posible asociación entre dos actividades: una investigó el reconocimiento de la aleatoriedad en tres situaciones de incertidumbre ambientadas en contextos diferentes (pensamiento, juego de azar y clima) y la otra, planteó la identificación de variables ocultas para explicar la vinculación contingente entre dos variables presentadas. Participaron en el estudio 218 jóvenes ingresantes a la Universidad seleccionados por accesibilidad. A partir de la categorización de las respuestas resultó que menos de un tercio de los participantes atribuyeron a la existencia de variables ocultas la aparente vinculación entre las variables presentadas mientras que el reconocimiento del azar dependió fuertemente del contexto de la situación planteada. Se efectuaron asociaciones entre las dos actividades, no encontrándose asociación entre la identificación de variables ocultas y el reconocimiento de la aleatoriedad para los ámbitos de juego de azar y del pensamiento, pero sí para el ámbito del clima.

Palabras claves: variable oculta - causalidad - aleatoriedad
\end{abstract}

\begin{abstract}
The possible association between two activities has been studied: one piece of research studied the recognition of random in three situations of uncertainty set in different contexts (thought, game of chance and climate), and the other studied the identification of hidden variables to explain the connection existing between two variables introduced. 218 young students about to be admitted to university which were selected by accessibility took part in this study. From the categorization of the answers the results showed that less than one third of the participants attributed the apparent connection between the variables introduced to the existence of hidden variables whereas the recognition of random depended strongly on the context of the given situation. Associations between the two activities were made, but no connections were found between identification of hidden variables and recognition of random for the setting of games of chance and thought, but they were found for the setting of climate.
\end{abstract}

Key words: hidden variable - causality - random

1 La investigación que se presenta en este artículo fue realizada con subsidios de la Universidad de Buenos Aires (UBACyT P043) y de la Agencia Nacional de Promoción Científica y Tecnológica (ANPCyT PICT 2004 N²0909)

2 Correspondencia: Horacio Félix Atoréis. Dirección Postal: Rivera Indarte 132 - $1^{\circ}$ A (1406) Buenos Aires - Argentina. E-mail: horacioattorresi@fibertel.com.arｈattorre@psi.uba.ar Teléfono: (5411) 4637-0923 
La noción de variable oculta se vincula a la de causalidad a través de disciplinas como la física, la filosofía y la psicología. Desde la filosofía, Leibniz planteó el principio de razón suficiente que sostiene que cada suceso tiene su causa en un suceso pasado. Así mismo, Kant sostuvo el principio de causalidad universal como soporte fundamental del pensamiento y afirmó que nuestra experiencia se rige por las nociones de causa y efecto. Algunos filósofos sostuvieron la causalidad universal como una necesidad metodológica, en el sentido de que cada suceso es tal que tiene garantizada su ocurrencia en referencia a algún suceso pasado. Arguyen que si ocurriera algún suceso para el que no fuese posible encontrar uno anterior responsable en términos causales, valdría preguntarse si se estudió el asunto el tiempo suficiente o exhaustivamente como para afirmar que no existe un suceso explicativo previo. En todo caso la búsqueda de dicho suceso explicativo depende de nuestra capacidad, posibilidades, limitaciones, etc. para encontrarlo (Sklar, 1994). Este último argumento ha sido un pilar sobre el que se sustenta el determinismo y que se vio conmocionado con el desarrollo en física de la teoría cuántica que negó la existencia necesaria de tal suceso explicativo.

En el estudio evolutivo del hombre se observa la adquisición de la causalidad desde los primeros meses de vida. Lamb (1981) expone la relación de contingencia tensión-alivio en niños de 2 a 6 meses de vida que le permite obtener, a través del llanto, una determinada conducta de los padres. Este mismo autor plantea que alrededor de los 6 meses, el niño comienza a diferenciar entre medios y fines, marcando la continuación del aprendizaje.

El estudio de las relaciones entre observaciones es un tema del que se ocupa la Estadística y es de gran utilidad en Psicología, no para hacer previsiones seguras sino más bien para juzgar la posibilidad de ocurrencia de dos sucesos sin que se establezca entre ellos una relación que implique causalidad. Suelen emplearse diferentes términos para hacer referencia a la ocurrencia de observaciones de al menos dos variables: no-independencia, asociación, relación estocástica, correlación, dependiendo de las técnicas estadísticas utilizadas sin que haya una regla estricta para su uso. No obstante, se reserva la noción de correlación para las escalas ordinales y especialmente para las de intervalos (Reuchlin, 1980).

Las personas pueden asumir juicios erróneos cuando no interpretan correctamente las variaciones conjuntas existentes entre variables. Existen técnicas de correlación que permiten determinar asociaciones entre variables, pero si esta relación es encontrada, no se puede asegurar que exista entre ellas una conexión causal. Existen diversos tipos de relaciones diferentes de la causal que pueden indicar la existencia de correlaciones entre variables. Por ejemplo, interdependencia, correlación espuria, dependencia indirecta, etc. (Estepa, 1995). Encontrar una relación estadísticamente significativa no es una condición suficiente para que esas variables se encuentren causalmente relacionadas. La correlación nos indica la ocurrencia conjunta de dichos acontecimientos, pero la vinculación entre ellos puede estar mediada por una variable que no es perceptible en el trabajo, es decir que puede existir una variable oculta que sea la responsable de la vinculación de los acontecimientos. Pérez Echeverría (1988) plantea que las personas tienen facilidades para percibir correlaciones aún donde no hay evidencia empírica. En ocasiones los individuos realizan una "correlación ilusoria”, que es la que se percibe bajo nuestras propias teorías, sin una base empírica. Chapman y Chapman (1969) presentaron esta correlación ilusoria como una idea de la sobrestimación que realizan los sujetos sobre la vinculación causal que existe entre la ocurrencia de dos sucesos que se hallan asociados conceptualmente. Por ejemplo, en correlaciones que realizan algunos individuos, incluso entrenados estadísticamente, sobre las características de los dibujos de los pacientes y ciertos rasgos psicológicos. Chapman y Chapman incluso cuestionan ciertas vinculaciones entre características del test de Rorschach y algunos síntomas patológicos.

En algunas circunstancias donde se interroga a los individuos sobre su opinión sobre la asociación entre dos acontecimientos, la pregunta que se formula, no es escueta, sino que está precedida por una cierta historia. De esta manera, no se brinda una confirmación total pero se construye un escenario, que le otorga mayor verosimilitud a la posible asociación (de Vega, 1995). Estos escenarios son imágenes que se obtienen por medio de combinar la intuición con el análisis y se basan en historias, relatos, que son verdaderos o creíbles. 
Tversky y Kahneman (1974) toman la problemática de la correlación ilusoria como una forma de manifestación del heurístico de accesibilidad. Además, estos autores presentan el heurístico de simulación cuando se construyen escenarios imaginarios, pues consideran este hecho como una herramienta cognitiva que puede llevar a errores debido a la simplicidad a la que se ha reducido una realidad compleja.

Cuando se plantean problemas formales (ej. extracción de bolillas) o relacionados con los juegos de azar puede ser que los individuos realicen mejores pronósticos debido a la representación mental del espacio muestral que pueden llevar a cabo. En cambio, la construcción de escenarios está vinculada con el ámbito social, donde es más difícil especificar este espacio muestral, por tal circunstancia es más visible la utilización de heurísticos como los de representatividad y accesibilidad para la toma de decisiones (de Vega, 1995).

Los individuos toman las variaciones conjuntas encontradas e introducen conceptualizaciones teóricas causales para producir el pasaje inferencial hacia la causalidad. Esta combinación le otorga a la causalidad una estructura constructiva que le permite generar explicaciones deterministas que resultan más plausibles que las no causales.

Ante un contexto cambiante, lleno de incertidumbre, la imputación de causas a las asociaciones encontradas parecería ser una manera de lograr el control de las situaciones, transformando el mundo en un lugar predecible. Esto puede llevar a las personas a esforzarse a encontrar relaciones causales, manipulando la información disponible.

La noción de azar está fuertemente ligada a la de imprevisibilidad y como tal suele ser concebida como una secuencia irregular de sucesos sin pautas o regularidades que se presentan en la vida cotidiana cuando nos enfrentamos con riesgos, predicciones, decisiones, etc. Estas situaciones suponen, a menudo, una limitación de la mente humana para abarcar lo complejo y lo incierto (Simon, 1955,1956). Desde un punto de vista psicológico, la noción de azar es extraña al comportamiento humano, ya que el pensamiento está orientado hacia una búsqueda de significado y se ocupa de dar sentido a la existencia, tanto en el mundo exterior como en la experiencia privada (Cohen, 1964). La incertidumbre no es fácilmente soportable y requiere de un esfuerzo psíquico que resulta superador para el desarrollo intelectual.

Las nociones de aleatoriedad y determinismo presentan coincidencias y diferencias que no son fáciles de señalar. En ocasiones, el azar es interpretado como la negación del determinismo y cercano a un pensamiento totalmente caótico, en otras, como algo tan evidente que no es analizado en mayor detalle. Frecuentemente se vinculan experiencias en las que interviene el azar con la noción de equiprobabilidad que remite a situaciones de máxima entropía, confundiéndose en estos casos la incertidumbre para la predicción de un resultado azaroso con la imposibilidad de llevar a cabo estrategias racionales de decisión.

En el ámbito de los juegos de azar, donde el reconocimiento de la aleatoriedad es alto, los individuos presentan dificultades para reconocer que dichos juegos siguen leyes estadísticas, a pesar de estar familiarizados con ellos desde edades muy tempranas. Se han encontrado argumentaciones vinculadas a lo mágico, a la superstición para explicar o predecir resultados posibles en una situación de incertidumbre, dependiendo dichas argumentaciones de la significatividad del estímulo presentado (Attorresi, García Díaz \& Pralong, 2007).

La comprensión y reconocimiento de los sucesos aleatorios son la base fundamental para el dominio de conceptos probabilísticos. Al estudiar la noción de imprevisibilidad desde el punto de vista de la comprensión se distinguen dos concepciones diferentes del azar. Una de ellas se basa en una idea de normatividad ante lo incierto que se rige por leyes y pautas y permite hacer predicciones respecto de situaciones aleatorias. La otra, basada en una idea no normativa de lo incierto, vincula al azar con lo caótico desprovisto de regularidades (Attorresi, García Díaz \& Pralong, 2005). El concepto de probabilidad se introduce cuando se buscan patrones de regularidad en los fenómenos aleatorios, es decir en aquellos experimentos en los que no se puede predecir el resultado. La pro- 
babilidad se concibe como la medida de la incertidumbre acerca de la aparición de un determinado evento cuando se realiza un experimento aleatorio (Bennett, 2000; Espino Morales, 2004).

Bajo el concepto de aleatoriedad se engloban un conjunto de términos tales como suceso, evento, etc, que se adoptan en distintas situaciones (Konold, 1991; Konold et al, 1991) y que se vinculan con argumentaciones basadas en la equiprobabilidad, múltiples posibilidades, incertidumbre y causalidad (Konold y Falk, 1992).

Tanur, Mosteller y Kruskal (1978) categorizan los fenómenos del azar en cuatro ámbitos: el psicológico-biológico, el físico-meteorológico, el social y el político.

En el presente trabajo se estudió la posible asociación entre dos tipos de actividades. En una de ellas se indagó el reconocimiento de la aleatoriedad al presentarse situaciones en las que interviene el azar. En la otra, se analizó la identificación de variables ocultas al plantearse la relación entre dos variables, problema en el que no interviene la noción de azar, sino que se trata de explicar la vinculación contingente entre dos variables presentadas. Esta última situación no supone un proceso aleatorio explícito, aunque para su tratamiento se recurra a métodos estadísticos, como lo son las pruebas de asociación entre variables, usuales para el estudio de fenómenos aleatorios. En base a las características mencionadas para las actividades que se presentan en este trabajo, es razonable formular la hipótesis de que no es esperable que exista asociación significativa entre ellas.

\section{Método}

\section{Diseño de Actividades}

Se diseñaron dos reactivos, el primero de ellos se utilizó para indagar el reconocimiento de variables ocultas al plantear la existencia de relación entre dos variables. Con este fin, se planteó una situación de contenido neutro para minimizar posibles distorsiones y sesgos. Se buscó una situación en la cuál no se descartara la relación entre las variables presentadas por una vinculación de contenido absurdo entre ellas.

Reactivo diseñado para el Reconocimiento de Variables Ocultas. (RVO)

En el área de Psicología Comunitaria un grupo de psicólogos ha investigado que en las localidades en las que los adolescentes pasan muchas horas frente a la televisión se ha registrado una alta proporción de cuadros de depresión. ¿Podrían interpretarse estos resultados como que mirar mucho tiempo la televisión puede causar cuadros de depresión? Explicá por qué.

El segundo reactivo fue diseñado previamente y utilizado para examinar el reconocimiento de hechos aleatorios con distintos contenidos y en distintos contextos en un trabajo previo (Attorresi, García Díaz \& Pralong, 2005). Dicho reactivo estaba compuesto de tres situaciones y a cada una de ellas los sujetos debían caracterizarla como aleatoria o no, fundamentando las respuestas. La primera corresponde al ámbito psicológico-biológico y estaba referida al pensamiento; la segunda con un perfil cotidiano-lúdico referida a un juego de azar, englobaba los ámbitos social y político; la tercera se construyó en un contexto físico-meteorológico.

Reactivo diseñado para el Reconocimiento de la Aleatoriedad (RA).

¿Cuál o cuáles de los siguientes sucesos pensás que es aleatorio? Justificá en cada caso por qué lo considerás aleatorio o no aleatorio.

- Ítem 1: La idea o pensamiento que tendrás en mente mañana a esta hora.

- Ítem 2: La cantidad de veces que tirás una moneda hasta que sale cara por primera vez.

- Ítem 3: Que llueva dentro de un mes. 
Se categorizaron las respuestas obtenidas para el reactivo diseñado RVO y se retomó la categorización básica para el reactivo compuesto RA.

Se realizaron Pruebas de Independencia Chi-cuadrado entre los reactivos.

\section{Participantes}

Se administró el reactivo a 218 jóvenes ingresantes a la Universidad de Buenos Aires, sin conocimientos previos acerca de probabilidades, provenientes de colegios públicos y privados de nivel socioeconómico medio. La muestra fue seleccionada por accesibilidad.

\section{Resultados}

A continuación se muestra las respuestas brindadas por los estudiantes para el reactivo RVO reunidas en tres grupos. En cada grupo se efectuó una subcategorización de los argumentos presentados.

Tabla1

\begin{tabular}{|c|c|c|}
\hline & & $\begin{array}{l}\text { Totales } \\
\mathrm{n}=218\end{array}$ \\
\hline \multirow{3}{*}{ 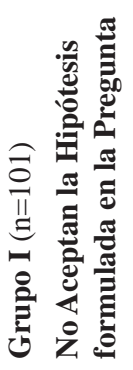 } & 1-Distinguen otros factores (variable oculta) & 63 \\
\hline & 2-Proponen un mejor estudio del fenómeno & 21 \\
\hline & 3-Le parece que no es cierto & 17 \\
\hline \multirow{4}{*}{ 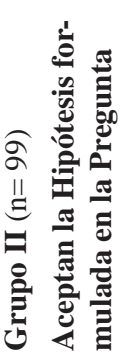 } & 1-Piensan que puede ser por casualidad & 11 \\
\hline & $\begin{array}{l}\text { 2-Distinguen otros factores vinculados o no vinculados a las horas } \\
\text { frente al televisor }\end{array}$ & 49 \\
\hline & 3-Da crédito a la afirmación por basarse en metodología científica & 17 \\
\hline & 4-Trata de justificar lo afirmado & 22 \\
\hline \multirow{2}{*}{ 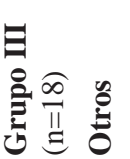 } & 1-Da respuesta y no justifica & 7 \\
\hline & 2-No contesta & 11 \\
\hline
\end{tabular}

En el Grupo I se reunieron las respuestas de quienes, correctamente, no aceptaron la hipótesis formulada en la pregunta. Dentro de este grupo se distinguieron las subcategorías siguientes:

I.1- Distinguen otros factores intervinientes en la relación entre las dos variables explicitadas, es decir, como es correcto, reconocen la existencia de variables ocultas que inciden en los resultados. Por ejemplo, el hecho de que los adolescentes que estén mucho tiempo solos o la ausencia de vínculos afectivos familiares o la falta de integración con sus pares o carecer de grupo de pertenencia, hace que se manifiesten en mayor medida cuadros depresivos. 
Otro ejemplo de variables ocultas intervinientes, relacionado con el contenido del reactivo presentado y referido a ciertas conductas adolescentes, es el fenómeno denominado en Japón hikikomoris, término que hace referencia al encierro que adoptan jóvenes, próximos a entrar en la vida adulta, que los mantiene confinados en sus habitaciones conectados a Internet frente a una pantalla y en las cuales permanecen días, meses e incluso años, evadiendo de esta forma las responsabilidades y las exigencias competitivas, laborales de la vida diaria. Algunos autores afirman que se trata de detener el tiempo y limitar el espacio en una especie de espacio/tiempo ficticio creado en la propia habitación (Feixa P`ampols, 2005).

I.2- Proponen un mejor estudio del fenómeno, ya que con la información que les es brindada sólo se deciden a negar la hipótesis.

I.3- Les parece que no es cierto, nuevamente sin dar mayores argumentos pero evidenciando intuitivamente desconfianza hacia lo sostenido en la hipótesis.

En el Grupo II se reunieron las respuestas de quienes erróneamente aceptaron la hipótesis formulada en la pregunta, distinguiéndose las siguientes subcategorías:

II.1- Piensan que la vinculación mencionada puede ser por casualidad, evidenciando dudas sobre la relación causal, aunque eso no impidió que dieran una respuesta afirmativa.

II.2- Distinguen otros factores vinculados o no a la cantidad de horas mirando televisión pero a diferencia del Grupo I argumentan que estos factores inciden favoreciendo la relación explicitada.

II.3- Dan crédito a la afirmación por basarse la situación presentada en una investigación científica.

II.4- Tratan de justificar la afirmación implícita incluso con argumentos forzados.

Un 53\% (101/218) de los consultados (Grupo I) no aceptaron la hipótesis formulada en la pregunta, negando en distinto grado la relación causal entre las variables presentadas. Las respuestas correctas correspondientes a quienes distinguieron otros factores que vinculaban a las variables (categoría I.1) fueron mayoría dentro de este grupo, 62\% (63/101), representando un 29\% (63/218) respecto del total de participantes.

Respecto del grupo que erróneamente aceptó la hipótesis formulada en la pregunta (Grupo II), que constituyeron el 45\% (99/218) del total de participantes, la mayoría un 49\% (49/99) brindó las mismas justificaciones que el grupo anterior, es decir, la existencia de otros factores vinculados o no al hecho de mirar televisión en forma prolongada (categoría II.2) pero que curiosamente los llevó a sostener una causalidad forzada.

Para el reactivo compuesto diseñado previamente para el reconocimiento de la aleatoriedad (RA) se retoma la categorización básica (Attorresi et al, 2005) de reconocimiento o no de la aleatoriedad de la situación presentada para el ámbito psicológico-biológico referido al pensamiento (RA, Item 1), para el ámbito social referido al juego de azar (RA, Item 2) y para el ámbito físico-meteorológico referido al clima (RA, Item 3). La categoría Otros reúne las respuestas de quienes no comprendieron la situación presentada o no justificaron o no contestaron.

Clasificación Básica para el Reconocimiento de la Aleatoriedad. 
Tabla 2

\begin{tabular}{|c|c|c|c|c|c|}
\hline & $\begin{array}{l}\text { Item } 1 \\
\mathrm{n}=218 \\
\text { Ámbito Psicológico-Bio- } \\
\text { lógico referido al pensa- } \\
\text { miento }\end{array}$ & \multicolumn{2}{|c|}{$\begin{array}{l}\text { Item } 2 \\
\text { n= } 218 \\
\text { Ámbito Social referido a } \\
\text { un juego de azar }\end{array}$} & \multicolumn{2}{|c|}{$\begin{array}{l}\text { Item } 3 \\
\mathrm{n}=218 \\
\text { Ámbito Físico-Meteoro- } \\
\text { lógico referido al clima }\end{array}$} \\
\hline $\begin{array}{l}\text { Reconocen Aleato- } \\
\text { riedad }\end{array}$ & $45 \%$ & 187 & $86 \%$ & 84 & $39 \%$ \\
\hline $\begin{array}{l}\text { No Reconocen Alea- } \\
\text { toriedad }\end{array}$ & $44 \%$ & 11 & $5 \%$ & 109 & $50 \%$ \\
\hline Otros & $11 \%$ & 20 & $9 \%$ & 25 & $11 \%$ \\
\hline
\end{tabular}

Como ya se señaló en el trabajo antes citado y se observa en la Tabla 2, los juegos de azar enmarcados en el ámbito social, son ampliamente reconocidos como manifestaciones típicas de lo aleatorio. En menor medida se le atribuye cierto grado de aleatoriedad a la idea fugaz, al pensamiento, correspondiente al ámbito psicológico-biológico y menor grado aún de aleatoriedad se asocia al clima correspondiente al ámbito físico- meteorológico, para el que los métodos científico-técnicos brindan conocimientos que parecerían aumentar el grado de predictibilidad y por lo tanto reducir la incertidumbre vinculada al azar.

\section{Pruebas de Independencia Chi-cuadrado entre reactivos}

Se estudió en forma conjunta el grado de reconocimiento del azar (reactivo RA) con la identificación de variables ocultas (reactivo RVO) como se muestra en la Tabla 3. Para el RA se agruparon los protocolos en tres categorías, bajo reconocimiento (los que reconocieron como azarosas ninguna o una de las situaciones presentadas), reconocimiento medio (los que reconocieron exactamente dos de las situaciones aleatorias presentadas) y alto reconocimiento (los que reconocieron las tres situaciones presentadas). Para el RVO se tuvieron en cuenta a los Grupos I (identificación de variables ocultas - sin relación causal) y II (no identificación de variables ocultas - causalidad forzada). Para ambos reactivos se eliminaron los protocolos de quienes respondieron sin justificar o no respondieron, es decir, aquellos agrupados en la categoría Otros de cualquiera de los dos reactivos (RVO y RA) conservándose los protocolos de 162 participantes. Se trabajó con un nivel de significación del $5 \%$.

Tabla 3

\begin{tabular}{|c|c|c|c|c|c|}
\hline \multirow[b]{2}{*}{ RVO } & & \multicolumn{3}{|c|}{ RA } & \\
\hline & & Bajo & Medio & Alto & \\
\hline \multirow[t]{3}{*}{ Grupo II } & Observ & 27 & 29 & 23 & 79 \\
\hline & Esperadas & 33.16 & 25.85 & 19.99 & \\
\hline & Chi-Cuad por celda| & 1.14 & 0.38 & 0.45 & \\
\hline \multirow[t]{5}{*}{ Grupo I } & Observ & 41 & 24 & 18 & 83 \\
\hline & Esperadas & 34.84 & 27.15 & 21.01 & \\
\hline & Chi-Cuad por celda| & 1.09 & 0.37 & $0.43 \mid$ & \\
\hline & & 68 & 53 & 41 & 162 \\
\hline & Chi-Cuadrado Total & 3.87 & P-Valor & 0.1446 & \\
\hline
\end{tabular}


La Tabla 3 muestra que no se puede rechazar la independencia entre los reactivos RA (reconocimiento del azar considerado globalmente en cuanto al grado) y RVO (identificación de variables ocultas). Este resultado era esperable si se considera la naturaleza diferente de los reactivos involucrados, que si bien son tratados con metodología estadística a través de las pruebas de independencia, uno de ellos, el RA, se enmarca en situaciones donde interviene el azar mientras que el otro, el RVO, no. El mismo resultado se obtiene si se estudia en forma conjunta el reconocimiento del azar en un ámbito determinado (RA Item 1 o RA Item 2) con la identificación de variables ocultas RVO como se muestra en las siguientes Tablas 4 y 5.

Tabla 4

RA Item 1

RVO

\section{Grupo II}

Grupo I
Observ

Esperadas

Chi-Cuad por celda|

Observ

Esperadas

Chi-Cuad por celda|

Chi-Cuadrado Total

\section{No Reconoce}

$\begin{array}{ccc}41 & 38 & 79 \\ 42.43 & 36.57 & \\ 0.05 & 0.06 & \\ 46 & 37 & \\ 44.57 & 38.43 & \\ 0.05 & 0.05 & \\ 87 & 75 & 162 \ldots \ldots \ldots \ldots \ldots \ldots \ldots \\ 0.20 & \text { P-Valor } & 0.6531\end{array}$

Tabla 5

RA Item 2

\begin{tabular}{|c|c|c|c|c|}
\hline RVO & & No Reconoce & Reconoce & \\
\hline \multirow[t]{3}{*}{ Grupo II } & Observ & 4 & 74 & 79 \\
\hline & Esperadas & 4.88 & 74.12 & \\
\hline & Chi-Cuad por celda| & 0.16 & 0.01 & \\
\hline \multirow[t]{5}{*}{ Grupo I } & Observ & 6 & 77 & 83 \\
\hline & Esperadas & 5.12 & 77.88 & \\
\hline & Chi-Cuad por celda| & 0.15 & 0.01 & \\
\hline & & 10 & 152 & 162 \\
\hline & Chi-Cuadrado Total & 0.33 & P-Valor & 0.5670 \\
\hline
\end{tabular}

El reconocimiento de la aleatoriedad fue destacado para el ámbito psicológico (RA Item 1) y muy alto para el ámbito social (RA Item 2) y para ambos contextos no mostró regularidad con el reconocimiento de variables ocultas, que supone muchos factores intervinientes aunque no explicitados.

El RA Item 3 fue considerado por los participantes como el menos sujeto a los efectos de la aleatoriedad, argumentándose que el desarrollo del conocimiento científico-técnico acerca de los fenómenos meteorológicos podía controlar dichos efectos. De este modo, se requirió de una consideración más cuidadosa y minuciosa acerca de la situación presentada para atribuirle cierto grado de incertidumbre. Se encontró una ligera dependencia entre el RA Item 3 y el RVO $(0.01<\mathrm{P}$-Valor $<0.05)$ como se muestra en la Tabla 6 . 
Tabla 6

RA Item 3

\begin{tabular}{|c|c|c|c|c|}
\hline RVO & & No Reconoce & Reconoce & \\
\hline \multirow[t]{3}{*}{ Grupo II } & Observ & 39 & 40 & 79 \\
\hline & Esperadas & 46.33 & 32.67 & \\
\hline & Chi-Cuad por celda| & 1.16 & 1.64 & \\
\hline \multirow[t]{5}{*}{ Grupo I } & Observ & 56 & 27 & 83 \\
\hline & Esperadas & 48.67 & 34.33 & \\
\hline & Chi-Cuad por celda| & 1.10 & 1.56 & \\
\hline & & 95 & 67 & 162 \\
\hline & Chi-Cuadrado Total & 5.47 & P-Valor & 0.0194 \\
\hline
\end{tabular}

El reconocimiento de la aleatoriedad planteado para el ámbito climático mostró un abanico de respuestas que apelaban a la causalidad y es probable que este hecho provocara la asociación con el reactivo RVO. Para este último las justificaciones relacionadas con el azar fueron escasas, apenas de un 5\% (11/218), correspondiendo a las respuestas que hicieron mención a la casualidad según se observa en la Tabla 1. En contraposición, en la identificación de variables ocultas existe una tendencia a buscar relaciones causales que se constituyen en eslabones que permiten vincular dos variables contingentes. Los factores causales presentes en los dos reactivos mencionados seguramente fueron los responsables de la asociación entre los mismos. En la Tabla 6 se observa que de los participantes que no reconocieron la aleatoriedad para el ámbito climático (95) la mayoría (56/95) sí identificó las variables ocultas para explicar la relación contingente entre las variables presentadas. En las justificaciones brindadas por este último grupo se advierte una tendencia de pensamiento histórico determinista. La idea de buscar factores no explícitos para la comprensión de situaciones más o menos complejas, buscando minimizar la incertidumbre presente en nuestro entorno, aunque se consiga con limitaciones, fue un substrato de los argumentos deterministas que tuvieron auge hasta principio del siglo XX, en contraposición a teorías que priorizaron lo aleatorio como un componente intrínseco de la naturaleza. Piénsese en la controversia Einstein- Bohr acerca del mundo de la microfísica. El primero apoyándose en la búsqueda de variables ocultas para la explicación de los fenómenos físicos y el segundo sosteniendo la presencia del azar y de la incertidumbre para la comprensión de dichos fenómenos.

\section{Discusión}

En el presente trabajo se advierte que menos de un tercio de los estudiantes identificaron la existencia de variables ocultas que vinculaban a las variables presentadas. El reconocimiento de otros factores intervinientes en dicha vinculación fue argumentado por aquellos participantes que entendieron adecuadamente la situación presentada. No obstante, la mención de otros factores intervinientes fue señalada, también, por quienes interpretaron erróneamente la situación, desestimando la importancia de estos otros factores a favor de una vinculación causal ficticia. Para este último grupo podría pensarse que el marco científico manifiesto en el contenido del reactivo perturbó el razonamiento de los consultados al conferirle ellos mismos a lo enunciado, llevado a cabo sobre dicho marco científico, un carácter de verdad indiscutible. Por otra parte, aquellos que rechazaron la hipótesis de vinculación causal planteada por la pregunta del reactivo, cuestión que es correcta, pero que no dieron justificaciones al respecto, parecerían responder a una especie de intuición donde se mezclan factores cognitivos y características de personalidad tales como la desconfianza respecto de lo enunciado o quien enuncia sin que se pueda establecer en qué proporción. Vale advertir que el no reconocimiento de variables ocultas o factores intervinientes no explícitos ante la posible relación entre dos variables puede llevar a establecer falsas causalidades y por lo tanto llevar a interpretacio- 
nes erróneas en los fenómenos de la vida diaria. Por otra parte el poder identificar variables ocultas constituye una de las bases sobre la que se construye la explicación de un fenómeno y su comprensión. Puede afirmarse entonces que los estudiantes que identificaron variables ocultas en la situación planteada (Grupo I) resultaron ser más explicativos.

Respecto del reconocimiento del azar se observó como éste depende fuertemente del contexto de la situación planteada, siendo el más favorable para dicho reconocimiento el de los juegos de azar (ámbito social) que el representado por la idea fugaz (ámbito psicológico-biológico) y más aun que el del clima (ámbito físico-meteorológico) lo cual marcó una diferencia en las asociaciones entre los reactivos.

Para la población involucrada, es decir, ingresantes a la Universidad sin formación sistemática en probabilidad, no se observó asociación entre el reconocimiento de la aleatoriedad considerado globalmente en cuanto al grado (RA) y la identificación de variables ocultas (RVO) como se esperaba dada la naturaleza conceptual diferente de ambas actividades. Tampoco se presentó asociación entre el reconocimiento de la aleatoriedad en el contexto lúdico RA Item 1 y en el del pensamiento RA Item 2 y la identificación de variables ocultas RVO. La única asociación encontrada fue entre este último reactivo RVO y el reconocimiento del azar para el contexto climático RA Item 3. Como ya se mencionó en los resultados, la búsqueda de relaciones causales que vinculen las variables presentadas para el reactivo RVO encontró asociación con la causalidad subyacente a las justificaciones que desestimaban la aleatoriedad del RA Item 3 apelando por ejemplo al desarrollo tecnológico o a la capacidad de predicción acerca de los fenómenos meteorológicos de la cual se dispone. El no reconocimiento de la aleatoriedad, en especial para el RA Item 3, da cuenta de un pensamiento más determinista que busca relaciones causales entre fenómenos y que se relaciona con la tendencia encontrada para la identificación de variables ocultas en cuanto a explicar la relación entre situaciones inconexas en torno a factores que median entre dichas situaciones.

En referencia a las controversias en la Física, antes mencionadas, de comienzos del siglo XX y de gran impacto en el pensamiento científico, se podría haberse supuesto la existencia de alguna relación inversa entre las habilidades estudiadas en los reactivos planteados. Una de ellas se refiere a la búsqueda de factores no explícitos que medien entre dos sucesos para la explicación de la ocurrencia de los mismos, indagada a través del reactivo RVO, en contraposición a una habilidad para reconocer las manifestaciones del azar en la vida cotidiana, presentes en el reactivo RA. Sin embargo, en general, dichas habilidades no están relacionadas en las prácticas educacionales. Si se tiene una consideración más detenida acerca de las situaciones presentadas, como se mencionó en la introducción, se trata de dos actividades de distinta naturaleza, ambas con posibilidades de ser estudiadas a través de la Estadística. En el RVO, sin embargo, no interviene conceptualmente el azar (como sí ocurre en el RA) sino más bien la construcción de la explicación a través de la búsqueda de variables ocultas.

\section{Referencias}

Attorresi H., García Díaz A. \& Pralong H. (2005). Identificación de situaciones aleatorias en estudiantes universitarios. Anuario de Investigaciones de la Facultad de Psicología. Universidad de Buenos Aires, 13-2,31-41.

Attorresi H., García Díaz A. \& Pralong H. (2007). Sesgos en la comprensión de dos situaciones típicas de incertidumbre y de azar. Perspectivas en Psicología. Universidad Nacional de Mar del Plata, 4-1,28-37.

Bennett, D. (2000). Aleatoriedad. Madrid. Alianza.

Cohen J. (1964). Azar, habilidad y suerte. Buenos Aires: Mirasol.

Chapman, L. J. y Chapman, J. P. (1969). Illusory correlation as an obstacle to the use of valid psychodiagnostic sign. Journal of Abnormal Psychology, 75, 271-280. 
de Vega, M. (1995). Introducción a la Psicología Cognitiva. Madrid: Alianza.

Espino Morales, O. (2004). Pensamiento y razonamiento. Madrid: Pirámide.

Estepa, A. (1995). Consideraciones sobre la enseñanza de la asociación estadística. UNO, 5, 69-79.

Feixa P`ampol, Carles (2005). Los hijos en casa: ¿hackers o hikikomoris? Revista Comunicación y Pedagogía. №208.

Konold, C. (1991). “Understanding Students’ Beliefs about Probability” en Glasesfeld E. von (ed.), Radical constructivism in Mathematics Educations. Dordrecht. Kluwer.

Konold, C., Lohmeier, J., Pollatsek, A., Well, A.D., Falk, R., and Lipson, A. (1991). “Novice Views on Randomness" en Proceedings of the Thirteenth Annual Meeting of the North American Chapter of the International Group for the Psychology of Mathematics Education, 1, ed. R.G. Underhill, Blacksburg. Virginia Polytechnic Institute and State University, pp. 167-173.

Konold, C. y Falk, R. (1992). "Encoding Difficulty: A Psychological Basis for "Misperceptions" of randomness" en The Sixteenth International Conference for Psychology of Mathematics Education. New Hampshire. Durham.

Lamb, M. E. (1981). The development of social expectations en the first year of life. En M. E. Lamb y L. R. Sherrod (Eds): Infant social cognition. Lawrence Erlbaum Associates, Publishers. Hillsdale, N. J.

Perez Echeverría, M. (1988). Psicología del Razonamiento Probabilística. Madrid: Universidad Autónoma.

Reuchlin, M. (1980). Compendio de Estadística. Madrid, Pablo del Río.

Simon, H. A. (1955). “A behavioral modelo f racional choice”. Quarterly

Journal of Economics, 1955, 69, 99-118.

Simon, H. A. (1956). Rational Choice and Structure of environment. Psychological Review, 1956, 63, 129-138.

Sklar, L (1994). Filosofía de la Física. Madrid: Alianza.

Tanur,J.; Mosteller, F.y Kruskal; W. (1978). Statistics: a guide to the unknown. California: HoldenDay.

Tversky, A. y Kahneman, D. (1974). Judment under uncertainty: Heuristics and biases. Science.185, 1124-1131. 